\title{
Computing Visual Correspondence with Occlusions using Graph Cuts
}

\author{
Vladimir Kolmogorov Ramin Zabih \\ Computer Science Department \\ Cornell University \\ Ithaca, NY 14853
}

\begin{abstract}
Several new algorithms for visual correspondence based on graph cuts [7, 14, 17] have recently been developed. While these methods give very strong results in practice, they do not handle occlusions properly. Specifically, they treat the two input images asymmetrically, and they do not ensure that a pixel corresponds to at most one pixel in the other image. In this paper, we present a new method which properly addresses occlusions, while preserving the advantages of graph cut algorithms. We give experimental results for stereo as well as motion, which demonstrate that our method performs well both at detecting occlusions and computing disparities.
\end{abstract}

\section{Introduction}

In the last few years, a new class of algorithms for visual correspondence has been developed that are based on graph cuts $[7,14,17]$. These methods give very strong experimental results; for example, a recent comparative study [18] of stereo algorithms found that one such algorithm gave the best overall results, with approximately 4 times fewer errors than standard methods such as normalized correlation. Unfortunately, existing graph cut algorithms do not treat occlusions correctly. In this paper, we present a new graph cut algorithm that handles occlusions properly, while maintaining the key advantages of graph cuts.

Occlusions are a major challenge for the accurate computation of visual correspondence. Occluded pixels are visible in only one image, so there is no corresponding pixel in the other image. For many applications, it is particularly important to obtain good results at discontinuities, which are places where occlusions often occur. Ideally, a pixel in one image should correspond to at most one pixel in the other image, and a pixel that corresponds to no pixel in the other image should be labeled as occluded. We will refer to this requirement as uniqueness.

Most algorithms for visual correspondence do not en- force uniqueness. (We will discuss algorithms that enforce uniqueness when we summarize related work in section 4). It is common to compute a disparity for each pixel in one (preferred) image. This treats the two images asymmetrically, and does not make full use of the information in both images. The recent algorithms based on graph cuts $[7,14,17]$ are typical in this regard, despite their strong performance in practice.

The new algorithm proposed in this paper is based on energy minimization. Our method is most closely related to the the expansion move algorithm of [8], which can find a strong local minimum of a natural class of energy functions. We address the correspondence problem by first constructing a problem representation and an energy function, such that a solution which violates uniqueness will have infinite energy. Constructing an appropriate energy function is non-trivial; for example, there are natural energy functions where it is NP-hard to even compute a local minimum. We then use graph cuts to compute a strong local minimum for our energy function.

This paper begins with a discussion of the expansion move algorithm of [8]. We then give an overview of our algorithm, in which we discuss our problem representation and our choice of energy function, and show how they enforce uniqueness. In section 4 we survey some related work, focusing on other algorithms that guarantee uniqueness. In section 5 we show how to compute a local minimum of our energy function in a strong sense using graph cuts. Experimental results are given in section 6. Detailed proofs are omitted to save space, but are contained in a technical report [13].

\section{Expansion moves}

Let $\mathcal{L}$ be the set of pixels in the left image, let $\mathcal{R}$ be the pixels in the right image, and let $\mathcal{P}$ be the set of all pixels: $\mathcal{P}=\mathcal{L} \cup \mathcal{R}$. The pixel $p$ will have coordinates $\left(p_{x}, p_{y}\right)$. In the classical approach to stereo, the goal is to compute, for each pixel in the left image, a label $f_{p}$ which denotes a disparity value for a pixel $p$. The energy minimized in [8] is 
the Potts energy ${ }^{1}$ of [16]

$$
E(f)=\sum_{p \in \mathcal{L}} D_{p}\left(f_{p}\right)+\sum_{p, q \in \mathcal{N}} V_{p, q} \cdot T\left(f_{p} \neq f_{q}\right)
$$

Here $D_{p}\left(f_{p}\right)$ is a penalty for the pixel $p$ to have the disparity $f_{p}, \mathcal{N}$ is a neighborhood system for the pixels of the left image and $T(\cdot)$ is 1 if its argument is true and 0 otherwise. Minimizing this energy is NP-complete, so [8] gives two approximation algorithms. They involve the notion of moves.

Consider a particular disparity (or label) $\alpha$. A configuration $f^{\prime}$ is said to be within a single $\alpha$-expansion move of $f$ if for all pixels $p \in \mathcal{L}$ either $f_{p}^{\prime}=f_{p}$ or $f_{p}^{\prime}=\alpha$. Now consider a pair of disparities $\alpha, \beta, \alpha \neq \beta$. A configuration $f^{\prime}$ is said to be within a single $\alpha \beta$-swap move of $f$ if for all pixels $p \in \mathcal{L}$ that had labels $\alpha$ or $\beta$ either $f_{p}^{\prime}=\alpha$ or $f_{p}^{\prime}=\beta$, and for all other pixels $f_{p}^{\prime}=f_{p}$.

The crucial fact about these moves is that for a given configuration $f$ it is possible to efficiently find a strong local minumum of the energy; more precisely, the lowest energy configuration within a single $\alpha$-expansion or $\alpha \beta$-swap move of $f$, respectively. These local improvement operations rely on graph cuts. The expansion algorithm consists entirely of a sequence of $\alpha$-expansion local improvement operations for different disparities $\alpha$, until no $\alpha$-expansion can reduce the energy. Similarly, the swap algorithm consists entirely of a sequence of $\alpha \beta$-swap local improvement operations for pairs of disparities $\alpha, \beta$, until no $\alpha \beta$-swap can reduce the energy.

This formulation, unfortunately, does not handle occlusions properly. First, two pixels in the left image can easily be mapped to the same pixel in the right image. Furthemore, the formulation assumes that each pixel in the left image is mapped into some pixel in the right image; in reality, some pixels in the left image can be occluded, and thus do not correspond to any pixel in the right image.

\section{Algorithm overview}

\subsection{Our representation}

Let $\mathcal{A}$ be the set of (unordered) pairs of pixels that may potentially correspond. For stereo with aligned cameras, for example, we have

$$
\mathcal{A}=\left\{\langle p, q\rangle \mid p_{y}=q_{y} \text { and } 0 \leq q_{x}-p_{x}<k\right\} .
$$

(Here we assume that disparities lie in some limited range, so each pixel in $\mathcal{L}$ can potentially correspond to one of $k$

\footnotetext{
${ }^{1}$ In fact, they consider a more general energy but this is the simplest case that works very well in practice.
}

possible pixels in $\mathcal{R}$, and vice versa). The situation for motion is similar, except that the set of possible disparities is 2-dimensional.

The goal is to find a subset of $\mathcal{A}$ containing only pairs of pixels which correspond to each other. Equivalently, we want to give each assignment $a \in \mathcal{A}$ a value $f_{a}$ which is 1 if the pixels $p$ and $q$ correspond, and otherwise 0 .

Let us define unique configurations $f$. We will call the assignments in $\mathcal{A}$ that have the value 1 active. Let $A(f)$ be the set of active assignments according to the configuration $f$. Let $N_{p}(f)$ be the set of active assignments in $f$ that involve the pixel $p$, i.e. $N_{p}(f)=\{\langle p, q\rangle \in A(f)\}$. We will call a configuration $f$ unique if each pixel is involved in at most one active assignment, i.e.

$$
\forall p \in \mathcal{P} \quad\left|N_{p}(f)\right| \leq 1 .
$$

Note that those pixels for which $\left|N_{p}(f)\right|=0$ are precisely the occluded pixels.

It is possible to extend the notion of $\alpha$-expansions for our representation. ${ }^{2}$ For an assignment $a=\langle p, q\rangle$ let $d(a)$ be its disparity: $d(a)=\left(q_{x}-p_{x}, q_{y}-p_{y}\right)$, and let $\mathcal{A}^{\alpha}$ be the set of all assignments in $\mathcal{A}$ having disparity $\alpha$. A configuration $f^{\prime}$ is said to be within a single $\alpha$-expansion move of $f$ if $A\left(f^{\prime}\right)$ is a subset of $A(f) \cup \mathcal{A}^{\alpha}$. In other words, some current active assignments may be deleted, and some assignments having disparity $\alpha$ may be added.

\subsection{Energy function}

Now we define the energy for a configuration $f$. To correctly handle unique configurations we assume that for nonunique configurations the energy is infinity and for unique configurations the energy is of the form

$$
E(f)=E_{\text {data }}(f)+E_{\text {occ }}(f)+E_{\text {smooth }}(f) .
$$

The three terms here include

- a data term $E_{\text {data }}$, which results from the differences in intensity between corresponding pixels;

- an occlusion term $E_{o c c}$, which imposes a penalty for making a pixel occluded; and

- a smoothness term $E_{\text {smooth }}$, which makes neighboring pixels in the same image tend to have similar disparities.

The data term will be $E_{\text {data }}(f)=\sum_{a \in A(f)} D(a)$; typically for an assignment $a=\langle p, q\rangle, D(a)=(I(p)-I(q))^{2}$, where $I$ gives the intensity of a pixel. The occlusion term

\footnotetext{
${ }^{2}$ It is also possible to extend the notion of an $\alpha \beta$-swap, as discussed in [13]. However, the resulting algorithm gives experimental results that are not as good as the current state of the art.
} 
imposes a penalty $C_{p}$ if the pixel $p$ is occluded; we will write this as

$$
E_{\text {occ }}(f)=\sum_{p \in \mathcal{P}} C_{p} \cdot T\left(\left|N_{p}(f)\right|=0\right),
$$

The most nontrivial part here is the choice of smoothness term. It is possible to write several expressions for the smoothness term. The smoothness term involves a notion of neighborhood; we assume that there is a neighborhood system on assignments

$$
\mathcal{N} \subset\{\{a 1, a 2\} \mid a 1, a 2 \in \mathcal{A})\} .
$$

One obvious choice is

$$
E_{\text {smooth }}(f)=\sum_{\{a 1, a 2\} \in \mathcal{N}, a 1, a 2 \in A(f)} V_{a 1, a 2},
$$

where the neighborhood system $\mathcal{N}$ consists only of pairs $\{a 1, a 2\}$ such that assignments $a 1$ and $a 2$ have different disparities (it can include, for example, pairs of assignments $\left\{\langle p, q\rangle,\left\langle p^{\prime}, q^{\prime}\right\rangle\right\}$ for which either $p$ and $p^{\prime}$ are neighbors or $q$ and $q^{\prime}$ are neighbors, and $\left.d(\langle p, q\rangle) \neq d\left(\left\langle p^{\prime}, q^{\prime}\right\rangle\right)\right)$. Thus, we impose a penalty if two close assignments having different disparities are both present in the configuration. Unfortunately, it can be shown that not only minimizing this energy is NP-complete, but also finding a minimum of this function among all configurations within a single $\alpha$-expansion of the initial configuration is NP-complete as well. (We give a simple reduction from the independent set problem to this problem in [13]).

We propose another smoothness term which makes it possible to use graph cuts to efficiently find a minimum of the energy among all configurations within a single $\alpha$ expansion of the initial configuration. The smoothness term will be

$$
E_{\text {smooth }}(f)=\sum_{\{a 1, a 2\} \in \mathcal{N}} V_{a 1, a 2} \cdot T(f(a 1) \neq f(a 2)) .
$$

The neighboorhood system here consists only of pairs $\{a 1, a 2\}$ such that assignments $a 1$ and $a 2$ have the same disparities (it can include, for example, pairs of assignments $\left\{\langle p, q\rangle,\left\langle p^{\prime}, q^{\prime}\right\rangle\right\}$ for which $p$ and $p^{\prime}$ are neighbors and $\left.d(\langle p, q\rangle)=d\left(\left\langle p^{\prime}, q^{\prime}\right\rangle\right)\right)$. Thus, we impose a penalty if one assignment is present in the configuration, and another close assignment having the same disparity is not. Although this energy is different from the previous one it enforces the same constraint: if adjacent pixels have the same disparity then the smoothness penalty is zero, otherwise it has some positive value.

The intuition why this energy allows using graph cuts is simply that it has a similar form to the Potts energy of equation 1. However, it is the Potts energy on assignments rather than pixels; as a consequence, none of the previous algorithms based on graph cuts can be applied.

\section{Related work}

Most work on motion and stereo does not explicitly consider occlusions. For example, correlation based approaches and energy minimization methods based on regularization [15] or Markov Random Fields [11] are typically formulated as labeling problems, where each pixel in one image must be assigned a disparity. This privileges one image over the other, and does not permit occlusions to be naturally incorporated. One common solution with correlation is called cross-checking [5]. This computes disparity twice, both left-to-right and right-to-left, and marks as occlusions those pixels in one image map to pixels in the other image which do not map back to them. This method is common and easy to implement, and we will do an experimental comparison against it in section 6.

Similarly, it is possible to incorporate occlusions into energy minimization methods by adding a label that represents being occluded. There are several difficulties, however. It is hard to design a natural energy function that incorporates this new label, and to impose the uniqueness constraint. In addition, these labeling problems still handle the input images asymmetrically.

However, there are a number of papers that elegantly handle occlusions in stereo using energy minimization [2, $4,10]$. These papers focus on computational modeling to understanding the psychophysics of stereopsis; in contrast, we are concerned with accurately computing disparity and occlusion for stereo and motion.

There is one major limitation of the algorithms proposed by $[2,4,10]$ which our work overcomes. These algorithms makes extensive use of the ordering constraint, which states that if an object is to the left of another in one stereo image, it is also to the left in the other image. The advantage of the ordering constraint is efficiency, as it permits the use of dynamic programming. However, the ordering constraint has several limitations. First, depending on the scene geometry, it is not always true. Second, the ordering constraint is specific to stereo, and cannot be used for motion. Third, algorithms that use the ordering constraint essentially solve the stereo problem independently for each scanline. While each scanline can be solved optimally, it is unclear how to impose some kind of inter-scanline consistency. Our method, in contrast, minimizes a natural 2-dimensional energy function, which can be applied to motion as well as to stereo.

Our algorithm is based on graph cuts, which can be used to efficiently minimize a wide range of energy functions. Originally, [12] proved that if there are only two labels the global minimum of the energy can be efficiently computed by a single graph cut. Recent work [7, 14, 17] has shown how to use graph cuts to handle more than two labels. The resulting algorithms have been applied to several problems in early vision, including image restoration and visual cor- 


\begin{tabular}{|c|c|c|c|}
\hline$p$ & $q$ & $r$ & $s$ \\
\hline & $y$ & & \\
\hline$w$ & $x$ & $y$ & $z$ \\
\hline
\end{tabular}

Figure 1. An example of two images with 4 pixels each. Here $\mathcal{L}=\{p, q, r, s\}$ and $\mathcal{R}=\{\mathrm{w}, \mathrm{x}, \mathrm{y}, \mathrm{z}\}$. Solid lines indicate the current active assignments, and dashed lines indicated the assignments being considered.

respondence. While graph cuts are a powerful optimization method, the methods of $[7,14,17]$ do not handle occlusions gracefully. In addition to all the difficulties just mentioned concerning occlusions and energy minimization, graph cut methods are only applicable to a limited set of energy functions. In particular, previous algorithms cannot be used to minimize the energy $E$ that we define in equation 2 .

The most closely related work consists of the recent algorithms based on graph cuts of [14] and [8]. These methods also cannot minimize our energy $E$. [14] uses graph cuts to explicitly handle occlusions. They handle the input images symetrically and enforce uniqueness. Their graph cut construction actually computes the global minimum in a single graph cut. The limitation of their work lies in the smoothness term, which is the $L_{1}$ distance. This smoothness term is not robust, and therefore does not produce good discontinuities. They prove that their construction is only applicable to convex (i.e., non-robust) smoothness terms. In addition, we can prove that minimizing our $E$ is NP-hard [13], so their construction clearly cannot be applied to our problem.

\section{Graph construction}

We now show how to efficiently minimize $E$ among all unique configurations using graph cuts. The output of our method will be a local minimum in a strong sense. In particular, consider an input configuration $f$ and a disparity $\alpha$. Another configuration $f^{\prime}$ is defined to be within a single $\alpha$ expansion of $f$ if some assignments in $f$ become inactive, and some assignments with disparity $\alpha$ become active (a formal definition is given at the start of section 5.2.1).

Our algorithm is very straightforward; we simply select (in a fixed order or at random) a disparity $\alpha$, and we find the unique configuration within a single $\alpha$-expansion move (our local improvement step). If this decreases the energy,

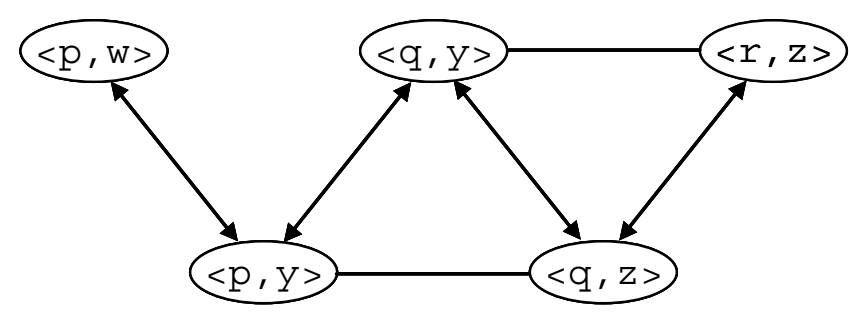

Figure 2. The graph corresponding to figure 1. There are links between all vertices and the terminals, which are not shown. Edges without arrows are bidirectional edges with the same weight in each direction; edges with arrows have different weights in each direction.

then we go there; if there is no $\alpha$ that decreases the energy, we are done. The critical step in our method is to efficiently compute the $\alpha$-expansion with the smallest energy. In this section, we show how to use graph cuts to solve this problem.

\subsection{Graph cuts}

Let $\mathcal{G}=\langle\mathcal{V}, \mathcal{E}\rangle$ be a weighted graph with two distinguished terminal vertices $\{s, t\}$ called the source and sink. A cut $\mathcal{C}=\mathcal{V}^{s}, \mathcal{V}^{t}$ is a partition of the vertices into two sets such that $s \in \mathcal{V}^{s}$ and $t \in \mathcal{V}^{t}{ }^{3}$ The cost of the cut, denoted $|\mathcal{C}|$, equals the sum of the weights of the edges between a vertex in $\mathcal{V}^{s}$ and a vertex in $\mathcal{V}^{t}$.

The minimum cut problem is to find the cut with the smallest cost. This problem can be solved very efficiently by computing the maximum flow between the terminals, according to a theorem due to Ford and Fulkerson [9]. There are a large number of fast algorithms for this problem (see [1], for example). The worst case complexity is low-order polynomial; however, in practice the running time is nearly linear for graphs with many short paths between the source and the sink, such as the one we will construct. In our current implementation we use a new max flow algorithm specifically designed for the kind of graphs that arise in energy minimization in vision [6].

\subsection{Computing a local minimum}

We first construct the graph $\mathcal{G}=\langle\mathcal{V}, \mathcal{E}\rangle$, and give the correspondence between cuts on $\mathcal{G}$ and configurations. Then we show that the minimum cut on $\mathcal{G}$ yields the configuration that minimizes $E$ among unique configurations within one $\alpha$-expansion.

\footnotetext{
${ }^{3} \mathrm{~A}$ cut can also be equivalently defined as the set of edges between the two sets.
} 


\subsubsection{Graph structure}

In an $\alpha$-expansion, active assignments may become inactive, and inactive assignments whose disparity is $\alpha$ may become active. Suppose that we start off with a unique configuration $f^{0}$. The active assignments for a new configuration within one $\alpha$-expansion will be a subset of $\tilde{A}=$ $\mathcal{A}^{0} \cup \mathcal{A}^{\alpha}$, where $\mathcal{A}^{0}=\left\{a \in A\left(f^{0}\right) \mid d(a) \neq \alpha\right\}$ and $\mathcal{A}^{\alpha}=\{a \in \mathcal{A} \mid d(a)=\alpha\}$. We will define the configuration $\tilde{f}$ by $A(\tilde{f})=\tilde{A}$. Note that in general $\tilde{f}$ is not unique.

The directed graph $\mathcal{G}$ that we will construct has vertices that correspond to assignments; this is in contrast to the graphs built by $[7,8,14,17]$. The terminals will be called $s$ and $t$, and for every assignment in $\tilde{A}$ there will be a vertex.

The edges in $\mathcal{G}$ are as follows. For every vertex $a \in$ $\tilde{A}$ there will be edges $(s, a)$ and $(a, t)$. In addition, if $\{a 1, a 2\} \in \mathcal{N}$ there will be edges $(a 1, a 2)$ and $(a 2, a 1)$. Note that in this case, either $a 1$ and $a 2$ are both in $\mathcal{A}^{0}$ or they are both in $\mathcal{A}^{\alpha}$. Finally, consider a pair of vertices $a 1, a 2$ that enter a common pixel $p$ (i.e., where $a 1=\langle p, q\rangle$ and $a 2=\langle p, r\rangle)$. Note that in this case either $a 1 \in \mathcal{A}^{0}, a 2 \in$ $\mathcal{A}^{\alpha}$ or vice-versa. There will be edges between every such pair of assignments.

Now consider a cut $\mathcal{C}=\mathcal{V}^{s}, \mathcal{V}^{t}$ on $\mathcal{G}$. The configuration $f^{\mathcal{C}}$ that corresponds to this cut is defined by

$$
\begin{aligned}
& \forall a \in \mathcal{A}^{0} \quad f_{a}^{\mathcal{C}}= \begin{cases}1 & \text { if } a \in \mathcal{V}^{s} \\
0 & \text { if } a \in \mathcal{V}^{t}\end{cases} \\
& \forall a \in \mathcal{A}^{\alpha} \quad f_{a}^{\mathcal{C}}= \begin{cases}1 & \text { if } a \in \mathcal{V}^{t} \\
0 & \text { if } a \in \mathcal{V}^{s}\end{cases}
\end{aligned}
$$

The following lemma is an obvious consequence of this construction.

Lemma $5.1 \mathcal{C}$ is a cut on $\mathcal{G}$ if and only if the configuration $f^{\mathcal{C}}$ lies within a single $\alpha$-expansion of the input configuration $f^{0}$.

We now give the weights of the edges in $\mathcal{G}$. First, we define the occlusion cost

$$
D_{o c c}(\langle p, q\rangle)=D_{o c c}(p)+D_{o c c}(q),
$$

where $D_{o c c}(p)=C_{p}$ if $\tilde{A}$ has only one edge entering $p$, and 0 otherwise. We define the smoothness cost by

$$
D_{\text {smooth }}(a 1)=\sum_{\substack{\{a 1, a 2\} \in \mathcal{N} \\ a 2 \notin \tilde{A}}} V_{a 1, a 2} .
$$

Then the weights are as follows.

\begin{tabular}{|c|c|c|}
\hline edge & weight & for \\
\hline \hline$(s, a)$ & $D_{o c c}(a)$ & $a \in \mathcal{A}^{0}$ \\
\hline$(a, t)$ & $D_{o c c}(a)$ & $a \in \mathcal{A}^{\alpha}$ \\
\hline$(a, t)$ & $D(a)+D_{\text {smooth }}(a)$ & $a \in \mathcal{A}^{0}$ \\
\hline$(s, a)$ & $D(a)$ & $a \in \mathcal{A}^{\alpha}$ \\
\hline$(a 1, a 2)$ & $V_{a 1, a 2}$ & $\begin{array}{c}\{a 1, a 2\} \in \mathcal{N}, \\
a 1, a 2 \in \tilde{A}\end{array}$ \\
\hline$(a 2, a 1)$ & $\infty$ & $\begin{array}{c}p \in \mathcal{P}, a 1 \in \mathcal{A}^{0}, a 2 \in \mathcal{A}^{\alpha} \\
a 1, a 2 \in N_{p}(\tilde{f})\end{array}$ \\
\hline$(a 2, a 1)$ & $C_{p}$ & $\begin{array}{c}p \in \mathcal{P}, a 1 \in \mathcal{A}^{0}, a 2 \in \mathcal{A}^{\alpha} \\
a 1, a 2 \in N_{p}(\tilde{f})\end{array}$ \\
\hline
\end{tabular}

We will refer to the links with weight $D_{o c c}(a)$ (i.e., the top two rows of the above table) as $t$-links. We will refer to the links with $\operatorname{cost} C_{p}$ as $c$-links.

A small example is shown in figure 1. The current set of assignments is shown with solid lines; dashed lines represent the new assignments we are considering (i.e., $\alpha=2$ ). In the current configuration, the pixels $\mathrm{s}$ and $\mathrm{x}$ are occluded, and the proposed expansion move will not change their status.

The corresponding graph is shown in figure 2 . The 3 nodes in the top row form $\mathcal{A}^{0}$ and the two nodes in the bottom row form $\mathcal{A}^{\alpha}$. Note, for example, that the edge from $\langle p, w\rangle$ to $\langle p, y\rangle$ has weight $\infty$, since these two assignments cannot both be active.

\subsubsection{Optimality}

We now show that if $\mathcal{C}$ is the minimum cut on our graph $\mathcal{G}$, then $f^{\mathcal{C}}$ is the configuration that minimizes the energy $E$ over unique configurations.

Lemma 5.2 The cost of the cut $\mathcal{C}$ is finite if and only if the corresponding configuration $f^{\mathcal{C}}$ is unique.

PRoOF: If $f^{\mathcal{C}}$ is not unique there is some pixel $p \in \mathcal{P}$ such that a pair of assignments $a 1, a 2 \in N_{p}\left(f^{\mathcal{C}}\right)$ are both in $A\left(f^{\mathcal{C}}\right)$. Without loss of generality let $a 1 \in \mathcal{A}^{0}$ and $a 2 \in$ $\mathcal{A}^{\alpha}$. Then we have $a 1 \in \mathcal{V}^{s}$ and $a 2 \in \mathcal{V}^{t}$, so the edge ( $a 1, a 2)$, which has weight $\infty$, must be cut. Similarly, if the weight of $\mathcal{C}$ is infinite, one of these edges is cut, so some pixel $p$ is not unique.

Lemma 5.3 Let $f^{\mathcal{C}}$ be a unique configuration, with corresponding cut $\mathcal{C}$. Then the cost of the t-links plus the c-links in $\mathcal{C}$ equals $E_{\text {occ }}\left(f^{\mathcal{C}}\right)$ plus a constant.

Theorem 5.4 Let $\mathcal{C}$ be the minimum cut on $\mathcal{G}$. Then $f^{\mathcal{C}}$ is the unique configuration within one $\alpha$-expansion of $f^{0}$ that minimizes the energy $E$. 
Due to space limitations, the proofs of lemma 5.3 and theorem 5.4 are included in [13].

\section{Experimental results}

Our experimental results involve both stereo and motion. Our optimization method does not have any parameters except for the exact choice of $E$. We selected the labels $\alpha$ in random order, and we started with an initial solution in which no assignments are active. For our data term $D$ we made use of the method of Birchfield and Tomasi [3] to handle sampling artifacts. The choice of $V_{a 1, a 2}$ was designed to make it more likely that a pair of adjacent pixels in one image with similar intensities would end up with similar disparities. If $a 1=\langle p, q\rangle$ and $a 2=\langle r, s\rangle$, then $V_{a 1, a 2}$ was implemented as an empirically selected decreasing function of $\max (|I(p)-I(r)|,|I(q)-I(s)|)$ as follows:

$$
V_{a 1, a 2}=\left\{\begin{array}{l}
\lambda \quad \text { if } \max (|I(p)-I(r)|,|I(q)-I(s)|)<8 \\
3 \lambda \quad \text { otherwise }
\end{array} .\right.
$$

The occlusion penalty was chosen to be $2.5 \lambda$ for all pixels. Thus, the energy depends only on one parameter $\lambda$. For different images we picked $\lambda$ empirically.

We compared our results with the expansion algorithm described in [8] with the explicit label 'occluded', since it is the closest related work. For the data with ground truth we obtained some recent results due to Zitnick and Kanade [20]. We also implemented correlation using the $L_{1}$ distance. Occlusions were computed using cross-checking, which computes matches left-to-right and right-to-left, and then marks a pixel as occluded if it maps to a pixel that does not map back to it. We used a 13 by 13 window for correlation; we experimented with several other window sizes and other variants of correlation, but they all gave comparable results.

Quantitative comparison of various methods was made on a stereo image pair from the University of Tsukuba with hand-labeled integer disparities. The left input image and the ground truth are shown in figure 3, together with our results and the results of various other methods. The Tsukuba images are 384 by 288 ; in all the experiments with this image pair we used 16 disparities.

We have computed the error statistics, which are shown in figure 4. We used the ground truth to determine which pixels are occluded. For the first two columns, we ignored the pixels that are occluded in the ground truth. We determined the percentage of the remaining pixels where the algorithm did not compute the correct disparity (the "Errors" column), or a disparity within \pm 1 of the correct disparity ("Gross errors"). We considered labeling a pixel as occluded to be a gross error. The last two columns show the error rates for occlusions.
In the electronic version of this paper, available from http://www.cs.cornell.edu/rdz, the occluded pixels for figures 3,5 and 6 are displayed in red. The running times for our algorithm are on average about $25 \%$ slower than the expansion algorithm of [8], but on the order of a minute. For example, on the Tsukuba data set our algorithm takes 83 seconds, while [8] takes 75 seconds. These numbers were obtained using a 500 Megahertz Pentium-III, and using the new max flow algorithm described in [6].

We have also experimented with the parameter sensitivity of our method. Since there is only one parameter, namely $\lambda$ in equation 4 , it is easy to experimentally determine the algorithm's sensitivity. The table below shows that our method is relatively insensitive to the exact choice of $\lambda$.

\begin{tabular}{l|llll}
$\lambda$ & 1 & 3 & 10 & 30 \\
\hline Error & $10.9 \%$ & $6.7 \%$ & $9.7 \%$ & $11.1 \%$ \\
Gross errors & $2.4 \%$ & $1.9 \%$ & $3.1 \%$ & $3.6 \%$ \\
False neg.'s & $42.2 \%$ & $42.6 \%$ & $48.0 \%$ & $51.4 \%$ \\
False pos.'s & $1.4 \%$ & $1.1 \%$ & $1.0 \%$ & $0.8 \%$
\end{tabular}

\section{Conclusions}

We have presented an energy minimization formulation of the correspondence problem with occlusions, and given a fast approximation algorithm based on graph cuts. The experimental results for both stereo and motion appear promising. Our method can easily be generalized to associate a cost with labeling a particular assignment as inactive.

\section{Acknowledgements}

We thank Y. Ohta and Y. Nakamura for supplying the ground truth imagery from the University of Tsukuba, L. Zitnick for providing us with the output of his method, and O. Veksler for useful comments. This research was supported by NSF grant IIS-9900115 and by a grant from Microsoft.

\section{References}

[1] Ravindra K. Ahuja, Thomas L. Magnanti, and James B. Orlin. Network Flows: Theory, Algorithms, and Applications. Prentice Hall, 1993.

[2] P.N. Belhumeur and D. Mumford. A Bayesian treatment of the stereo correspondence problem using halfoccluded regions. In IEEE Conference on Computer Vision and Pattern Recognition, pages 506-512, 1992. Revised version appears in IJCV.

[3] Stan Birchfield and Carlo Tomasi. A pixel dissimilarity measure that is insensitive to image sampling. 


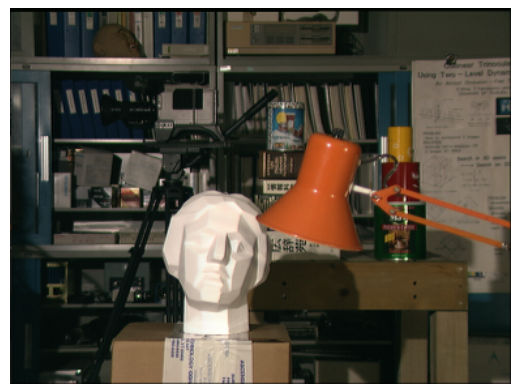

Left image

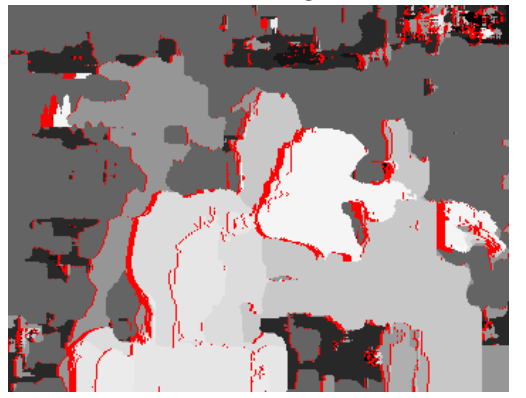

$L_{1}$ correlation

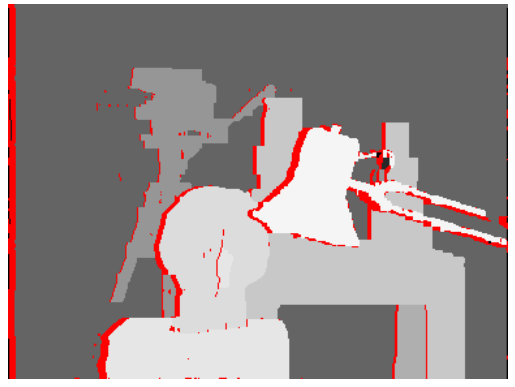

Our results

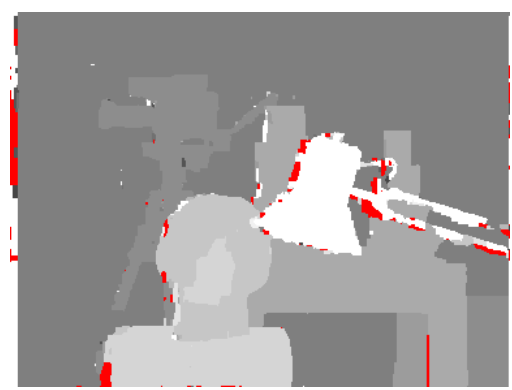

Results from [8]

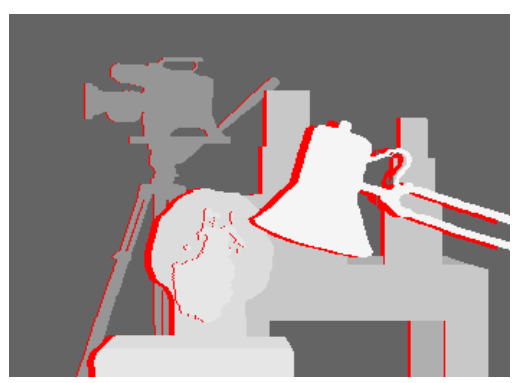

Ground truth

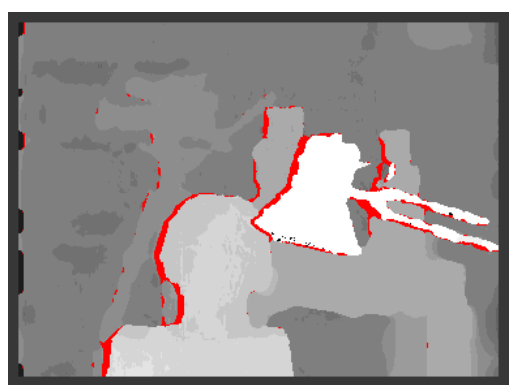

Results from [20]

Figure 3. Stereo results on Tsukuba dataset. Occluded pixels are shown in red in the electronic version of this paper.

\begin{tabular}{l|cccc} 
Method & Errors & Gross errors & False negatives & False positives \\
\hline Our results & $6.7 \%$ & $1.9 \%$ & $42.6 \%$ & $1.1 \%$ \\
Boykov, Veksler \& Zabih [8] & $6.7 \%$ & $2.0 \%$ & $82.8 \%$ & $0.3 \%$ \\
Zitnick \& Kanade [20] & $12.0 \%$ & $2.6 \%$ & $52.4 \%$ & $0.8 \%$ \\
Correlation & $28.5 \%$ & $12.8 \%$ & $87.3 \%$ & $6.1 \%$
\end{tabular}

Figure 4. Error statistics on Tsukuba dataset.

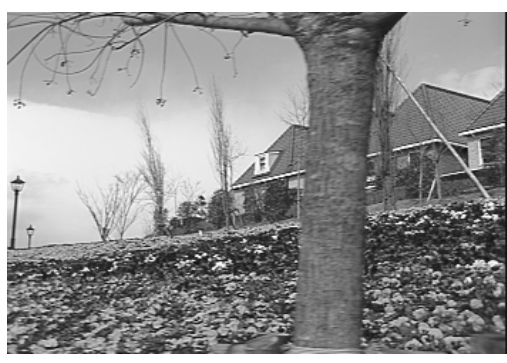

First image

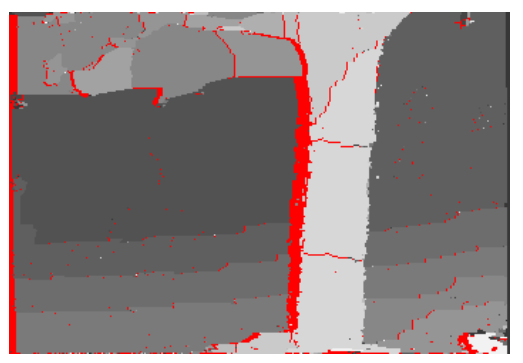

Horizontal motion

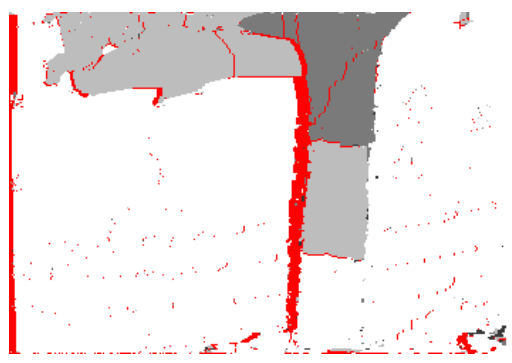

Vertical motion

Figure 5. Motion results on the flower garden sequence. Occluded pixels are shown in red in the electronic version of this paper. 


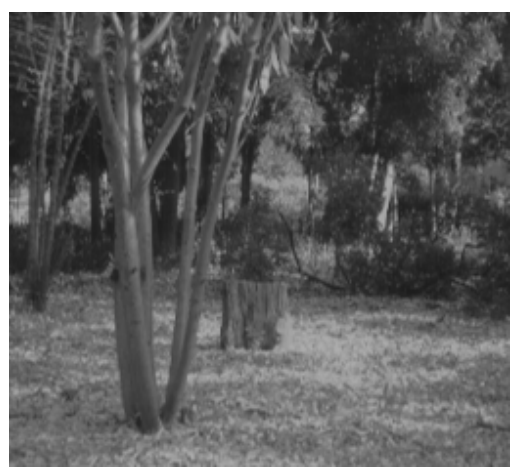

First image

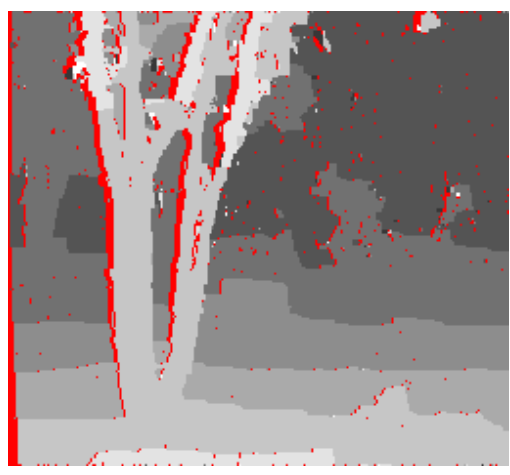

Horizontal motion (our method)

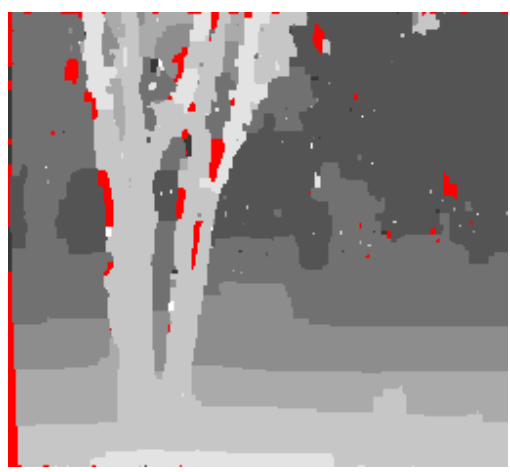

Horizontal motion (method of [8])

\section{Figure 6. Stereo results on the SRI tree sequence. Occluded pixels are shown in red in the electronic version of this paper.}

IEEE Transactions on Pattern Analysis and Machine Intelligence, 20(4):401-406, April 1998.

[4] A.F. Bobick and S.S. Intille. Large occlusion stereo. International Journal of Computer Vision, 33(3):1-20, September 1999.

[5] Robert C. Bolles and John Woodfill. Spatiotemporal consistency checking of passive range data. In International Symposium on Robotics Research, 1993.

[6] Yuri Boykov and Vladimir Kolmogorov. An Experimental Comparison of Min-Cut/Max-Flow Algorithms for Energy Minimization in Vision. In International Workshop on Energy Minimization Methods in Computer Vision and Pattern Recognition, 2001.

[7] Yuri Boykov, Olga Veksler, and Ramin Zabih. Markov random fields with efficient approximations. In IEEE Conference on Computer Vision and Pattern Recognition, pages 648-655, 1998.

[8] Yuri Boykov, Olga Veksler, and Ramin Zabih. Fast approximate energy minimization via graph cuts. In International Conference on Computer Vision, pages 377-384, September 1999.

[9] L. Ford and D. Fulkerson. Flows in Networks. Princeton University Press, 1962.

[10] D. Geiger, B. Ladendorf, and A. Yuille. Occlusions and binocular stereo. International Journal of Computer Vision, 14(3):211-226, April 1995.

[11] S. Geman and D. Geman. Stochastic relaxation, Gibbs distributions, and the Bayesian restoration of images. IEEE Transactions on Pattern Analysis and Machine Intelligence, 6:721-741, 1984.
[12] D. Greig, B. Porteous, and A. Seheult. Exact maximum a posteriori estimation for binary images. Journal of the Royal Statistical Society, Series B, 51(2):271-279, 1989.

[13] Vladimir Kolmogorov and Ramin Zabih Computing Visual Correspondence with Occlusions via Graph Cuts. Cornell CS technical report CUCS-TR-20011838, March 2001.

[14] H. Ishikawa and D. Geiger. Occlusions, discontinuities, and epipolar lines in stereo. In European Conference on Computer Vision, pages 232-248, 1998.

[15] Tomaso Poggio, Vincent Torre, and Christof Koch. Computational vision and regularization theory. $\mathrm{Na}$ ture, 317:314-319, 1985.

[16] R. Potts. Some generalized order-disorder transformation. Proceedings of the Cambridge Philosophical Society, 48:106-109, 1952.

[17] S. Roy. Stereo without epipolar lines: A maximum flow formulation. International Journal of Computer Vision, 1(2):1-15, 1999.

[18] R. Szeliski and R. Zabih. An experimental comparison of stereo algorithms. In B. Triggs, A. Zisserman, and R. Szeliski, editors, Vision Algorithms: Theory and Practice, number 1883 in LNCS, pages 1-19.

[19] Olga Veksler. Efficient Graph-based Energy Minimization Methods in Computer Vision. PhD thesis, Cornell University, August 1999.

[20] C. Lawrence Zitnick and Takeo Kanade. A cooperative algorithm for stereo matching and occlusion detection. IEEE Transactions on Pattern Analysis and Machine Intelligence, 22(7):675-684, July 2000. 